\title{
Transmission Electron Microscopy of Mn-doped KNN Ceramics
}

\author{
M.A Rafiq*, M.E. Costa**, I.M. Reaney** and P.M. Vilarinho* \\ *Department of Materials and Ceramic Engineering, Centre for Research in Ceramics and Composite Materials, \\ CICECO, University of Aveiro, 3810-193 Aveiro, Portugal \\ **Department of Materials Science and Engineering, University of Sheffield, Sheffield, S1 3JD, UK
}

Smart materials like piezoelectrics and ferroelectrics play a crucial role in applications such assensors and actuators,radio-frequency switching, drug delivery, chemicals detection, and power generation and storage. $\mathrm{K}_{0.5} \mathrm{Na}_{0.5} \mathrm{NbO}_{3}(\mathrm{KNN})$ is one of the leading lead free piezoelectric materials being considered as an alternativeto $\mathrm{Pb}\left(\mathrm{Zr}_{\mathrm{x}}, \mathrm{Ti}_{1-\mathrm{x}}\right) \mathrm{O}_{3}(\mathrm{PZT})$, which is currently the most widely used material for electromechanical applications[1]. Although pure KNN has inferior electromechanical properties compared to PZT,efforts are on going to tailor and improve its piezoelectric coefficients by doping and texturing [2,3].Although the piezoelectric constant $\left(\mathrm{d}_{33}\right)$ of undoped KNN is unsuitable for practical electromechanical applications, properties comparable to PZT at room temperature $\left(\mathrm{d}_{33}>400 \mathrm{pC} / \mathrm{N}\right)$ have been reported for modified KNN ceramics. Electromechanical properties are however, very much dependent on the crystalline phase content, crystallographic orientation, microstructure, interfaces and domain configuration.

$\mathrm{Mn}$ is an indispensable dopant for both $\mathrm{PbO}$-based as well as $\mathrm{PbO}$-free ceramics like $\mathrm{BaTiO}_{3}, \mathrm{SrTiO}_{3}, \mathrm{KNbO}_{3}$ and $\mathrm{KTaO}_{3}$. It has been reported to improve the density, mechanical quality factor, electromechanical properties and to reduce dielectric loss. Mn has been successfully used to reduce the leakage current and lower the orthorhombic to tetragonal phase transition temperature $\left(T_{O-T}\right)$ in KNN single crystals. It has also been shown to improve the density and properties of $\mathrm{KNN}-\mathrm{LiTaO}_{3}-\mathrm{LiSbO}_{3}$. However, the effect of $\mathrm{Mn}$ on the KNN domain structure and phase assemblage has not yet been reported. In this work, KNN ceramics doped with Mn on the Bsite (Mn content was $0.5,1.0 .1 .5$ and $2 \mathrm{~mole} \%$ ) were synthesized by a conventional mixed oxide method. Transmission electron microscopy (TEM)(Hitachi 9000) studies were carried out to analyse the effect of B-site Mn doping on the ferroelectric domain structure and phase assemblage.

Undoped KNN ceramics had large grains $(>30 \mu \mathrm{m})$ which contained large $(>1 \mu \mathrm{m}$ wide) wedge shaped ferroelectric domains. KNN doped with 0.5 mole $\% \mathrm{Mn}$ exhibited a smaller grain size $(\sim 2 \mu \mathrm{m})$ in which a well defined domain structure was observed with widths approximately an order of magnitude smaller than those in undoped KNN. For KNN doped with 2 mole \% Mn, the presence of a second phase, Figure 1c, was often observed. Electron diffraction patterns from the second phase were consistent with a tetragonal tungsten bronze (TTB) structured compound although more work is required to definitively determine the phase assemblage. The domain structure became increasing complex as Mn concentration increased, suggesting that the presence of $\mathrm{Mn}$ on the B-site disrupts polar order.

In conclusion, TEM analysis demonstrated that Mn doping changes the domain structure of KNN ceramics: for low Mn content, well defined ferroelectric domains and for high Mn content, tangled domains and second phase were the main features. These microstructure details elucidate reasons that may account for the inferior piezoelectric properties of KNN at higher Mn concentration. 


\section{References}

1. Saito Y. et al., Nature, 432:84, 2005.

2. Rodel J. et al., J. Am. Ceram. Soc., 92:1153, 2009.

3. Wang K. et al., Adv. Funct. Mater., 20:1924, 2010.
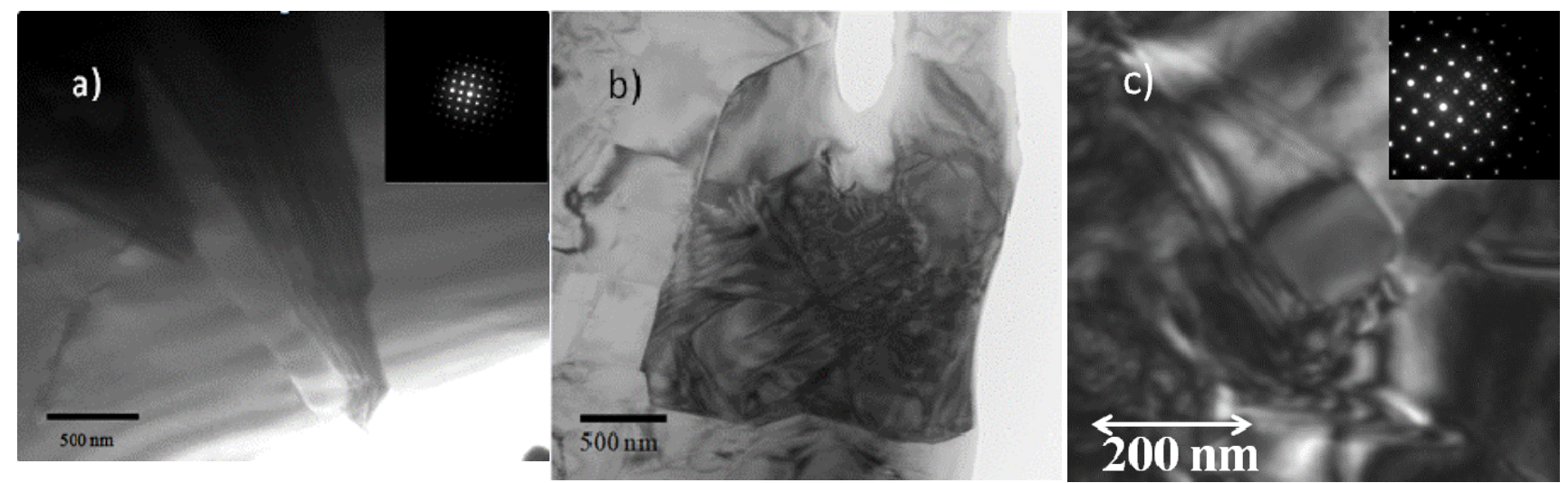

Figure 1. TEM micrographs of ferroelectric domains in KNN ceramics: (a) undoped with inset [001] diffraction pattern where $\mathrm{o}=$ orthorhombic (b) 0.5 mole $\%$ Mn doped with orthorhombic phase and (c) 2 mole \% Mn doped with tangled domain and a secondary phase precipitate. Inset in $\mathrm{c}$ ) is a $[001]_{\mathrm{o}} / /[001]_{\mathrm{t}}$ where $\mathrm{t}=$ tetragonal diffraction pattern obtained from both the matrix and precipitate. The precipitate pattern is consistent with a tetragonal tungsten bronze structured (TTB) structured compound. 